\title{
Diastolic Failure: Pathophysiology and Therapentic Implications
}

\author{
DIRK L. BRUTSAERT, MD, PHD, FACC, STANISLAS U. SYS, MD, THIERRY C. GILLEBERT, MD \\ Antwerp, Belgium
}

Primary diastolic dysfunction or failure is a distinet pathophysiologic entity. It results from increased resistance to ventricular filling, which leads to an inappropriate upward shift of the diastolic pressure-volume relation, particularly during exercise (exercise intolerance). The catses of diastolic failure are inappropriate tachycardia, decreased diastolic compliance and impaired systolic relaxation. Impaired (incomplete or slowed) systolic re. laxation must be conceptually distinguished from compensatory

prolonged systolic contraction (dilayed or retarded relaxation). Optimal therapy will depend on the type of disease, the phase during the course of a given disease and the coexistence and relative contribution of varioas (de)compensatory processes. Treatment may consist of bradycardic, remodeling and busitropic drugs.

(J Am Coll Cardiol 1993;22:318-25)

Diastolic failure of the heart is a widely recognized clinical entity. Whereas most cases of diastolic failure are secondary to systolic failure and are part of the terminal picture of congestive heart failure, there also exists a primary form of diastolic failure commonly defined as "a condition with classic findings of congestive failure with abnormal diastolic but normal systolic function at rest" (1-9). Because the first symptoms of congestive diastolic failure often occur during exercise, exercise intolerance is considered as an early sign of diastolic failure (10). It is observed as an early event in many clinical conditions, such as hypertrophic cardiomyopathy (including aortic stenosis and hypertensive heart disease) and ischemic cardiomyopathy. In this sense, diastolic dysfunction with manifest diastolic failure during exercise is more common than originally thougit (that is, approximately $40 \%$ of all conditions of congestive heart failure seem to evolve from diastolic failure). Nevertheless, isolated left ventricular diastolic failure or dysfunction has been shown to be associated with a low cardiac mortality rate; at the same time, it is associated with substantial morbidity (11).

Because there is often confusion about the use of the terms dicstole and diastolic function or dysfunction, one should first make clear what component or phase of the cardiac cycle is under consideration. We therefore first consider the term diastole and reexamine the definition of diastolic failure. We then review some of the known causes and discuss some therapeutic implications.

From the Department of Physiology and Medicine and University Hospital. Antwerp University, Antweip, Belgium.

Manuscript received July 22, 1992; revised manuscript received November 25, 1992, accepted December 1, 1992.

Address for comespondence: Dirk L. Brutsaert, MD, PhD, University of Antwerp, Groenenborgerlaan 171, 2020 Antwerp, Belgium.

\section{Definition of Diastole and Diastolic Failure}

The heart is a muscular pump. In the same way as in isolated cardiac muscle, a decrease in force and relengthening during relaxation of an afterloaded twitch are parts of one activity transient (that is, part of one contraction-relaxation cycle [or "systole"]), a decrease in ventricular pressure and an increase in ventricular volume during early rapid filling are closely related to this activity transient and should therefore be considered as part of systole (12). On these conceptual grounds, the term diastole should be restricted to the phase during the cardiac cycle that separates two such consecutive contraction-relaxation transients, that is, to the diastasis and the atrial contraction phase, or approximately the last $5 \%$ to $15 \%$ of volume change during cardiac filling on a pressure-volume diagram (Fig. 1, points 3 to 4).

The previous definition of diastolic failure sinould therefore be restated as follows: "a condition resulting from an increased resistance to filling of one or both ventricles, leading to symptoms of congestion due to an inappropriate upward shift of the diastolic pressure-volume relation (that is, during the terminal phase of the cardiac cycle). Whether this upward shift of the diastolic pressure-volume relation is caused by increased pressure for a given volume or by increased pressure inappropriate or disproportionate to the increase in volume or whether the shift is symmetric or asymmetric will not affect the hemodynamic consequences: pulmonary wedge pressure will increase (10). Accordingly, the upward shift of the diastolic presstre-volume relation may lead to symptoms of congestion, often at rest but particularly during exercise, manifesting as exercise intolerance. This shift may also lead to subendocardial ischemia and, if chronic, to remodeling and hypertrophy of the ventricle.

A common problem in science is the difficulty in formulating the appropriate questions. In the publishec repots on 


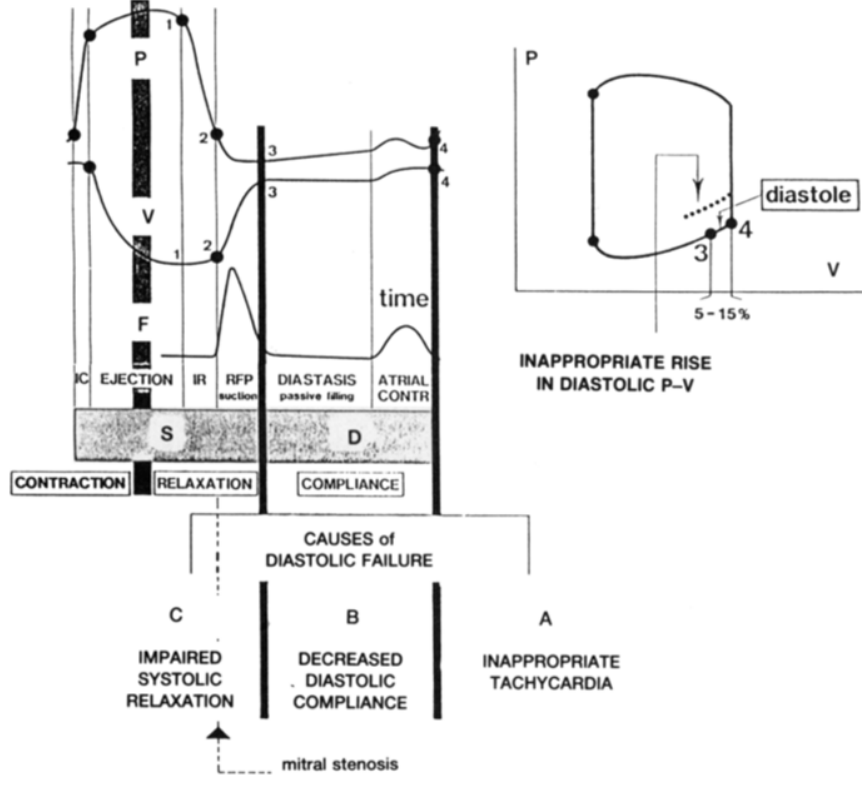

Pigure 1. Subdivision of the cardiac cycle into systole (S) and diastole (D), illustraing the causes of diastolic failure: CONTR = contraction: $\mathbf{F}=$ flow; $\mathbb{I C}=$ isovolumeiric contraction: $\mathbb{R}=$ isovolumetric relaxation; $\mathbb{P}=$ pressure; $R F P=$ rapid filling phase; $\mathrm{V}=$ volume. 1 = aortic valve closure; 2 = mitral valve opening; $3=$ end of early rapid filling; $4=$ end-diastole. Modified from Brutsaert and Sys (12).

primary diastolic failure, two basic questions have been asked interchangeably. 1) Is relaxation impaired before contraction abnormalities occur? 2) Is diastole imparred before systolic dysfunction occurs? These are obviously two different issues that, if not conceptually defined and separated, will inevitably lead to conflicting conclusions. Part of the problem is semantic because many clinicians still erroneously continue to consider the greater portion of ventricular relaxation as an integral part of diastole $(5,13-15)$.

Relazation versus contraction impaiment? Given the close conceptual link between contraction and relaxation (12), systolic dysfunction may be manifested as an abnormality of contraction or of relaxation, or of both. Depending on the underlying cellular mechanisms, relaxation may indeed seem to be impaired before contraction in many instances. For example, impairment of the late phase of ventricular relaxation, including rapid filling, which is predominantly caused by abnormalities in calcium handling, may be diagnosed by so-called late relaxation measurements, such as a prolongation of tau or a decreased rapid filling rate. If, however, only the very early time course of relaxation is impaired (for example, because of impaired contractile protein interaction and detachment), measurements that incorporate this early phase of relaxation, such as stroke work, ejection fraction, end-systolic pressure-volume relation. reak negacive first derivative of left re slar pressure (dP/dt) may be abnormal even in the presence of normal late relaxation indexes. In most clinical conditions, however, contraction and relaxation abnomalities are merely parts of one ccntinuous spectrum of systolic abnorzialities due either to impaired interaction and detachment of the contractile proteins or to abnormalities in the calcium handling, or both. An extensive discussion of these underlying cellular mechanisms has been published elsewhere (12).

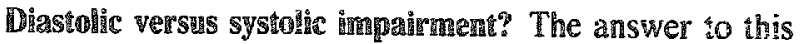
second question is lesミ clear and is still under debat: (16) because many clinical investigators continue to consider a major part of systolic relaxation, including isovolumenfic jressure decrease and early rapid filling, as an integral par.

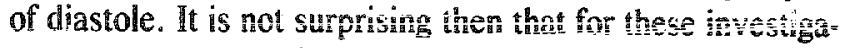
tors, the answer to the second question would, even in the presence of unchanged diastolic pressure-volume relations, parallel the answer to the first question-hence the several inconsistencies in published data. We now discuss how, on theoretic grounds, both genuine diastolic abnormalities and impared systolic relaxation may lead to diastolic failure, as restated earlier. Although the causes ar often multifactorial, diastolic failure may in specific cases occur in the absence of systolic (contraction or relaxation) abmormalites.

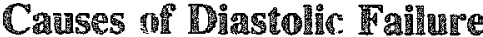

The three causes of an upward shift of the diastolic pressure-volume relation can be classified as follows (Fig. 1): The first relates to inappropriately high heart rates (not discussed further here); the second deals with fundaniental abnormalities of the myocardium at rest, wich directly result in decreased diastolic compliance; the third $\mathrm{ks}$ the indirect consequence of abnormalities during the second part of systole (that is, during systolic relaxation). Although all three causes could contribute separately to an upward shift of the diastolic pressure-volume relation, in mary conditions all three act in concert. Hence in many diseases, diastolic failure may have a multifactorial origin. (Mitral stenosis is not dealt with here because it constitutes a special case of diastolic failure. Although it is perhaps the best example provided by nature, mimicking the pathogenesis and symptoms of classic primary diastolic failure, the eliciting pathophysiology clearly does not comply with the previous definition of an inappropriate upward shift of the diastolic ventricular pressure-volume relation.)

Table I lists several possible causes of decreased diastolic ventricular compliance, some of which originate from geometric changes in the ventricular wall or from structural changes in muscle or in interstitial space. Other causes have an extraventricular origin (the pericardium, for example). A.part from direct compression of the ventricles during diastole in various pericardial diseases, the normal pericardium may, in addition, act as an external mectuanical con. straint, in particular during diastole in patients with cardiac dilation $(12,17-19)$. Here, even the sightest increase in pressure or volume of the right ventricle will increase pericardial pressure, which will be transmitted to the left ventricle, wherf: it will further increase the diastolic 
Table 1. Various Conditions of Decreased Diastolic Compliance as Causes of Diastolic Fóilure

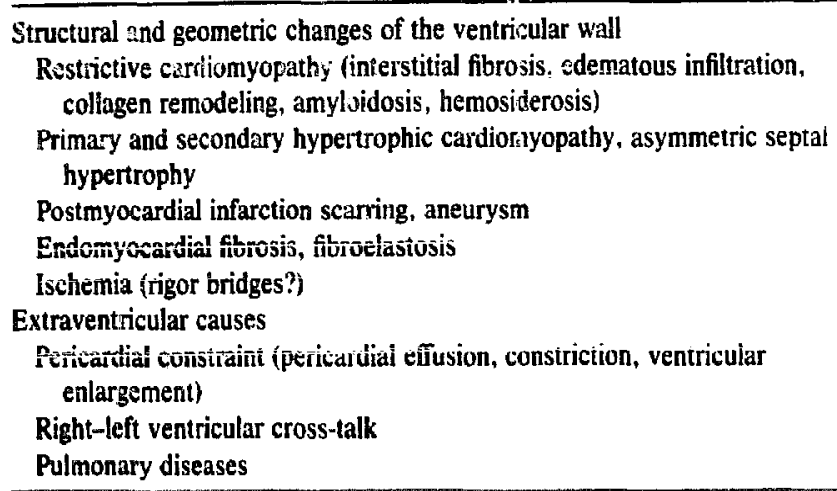

pressure-volume relation, with no direct repercussion on systolic function (17). Equally important and often related to conditions of pericardial constraint is the potential functional importance of right-left ventricular interdependence in conditions of acute unilateral right ventricular overload. The resulting "cross-talk" may have implications with respect to some more specific clinical conditions or to some forms of treatment; for example, pulmonary embolism or drug interventions that act on the loading conditions of the right ventricle may indirectly also affect measured left ventricular compliance during diastole.

A common cause of diastolic failure is impaired (or slowed or inccmplete) systolic relaxation of the left ventricle. Impaired ielaxaticn must be distinguished from delayed or retarded relaxation: the latter condition should preferably be called prolonged contraction (Fig. 2). Although both conditions are often trarely distinguishable in clinical cardiology, it is important to make the distinction on conceptual

Figure 2. Impaired syst Jlic relaxation (right) conpared with conditions of prolonged systolic contraction (ieft). Impaired systolic relaxation, but not prolonged contraction, can be a cause of diastolic failure. $a, b, c=$ without $(a)$ or with $(b, c)$ physiologic changes in rate of relaxation; $-d P / d t=$ peak negative first derivative of left ventricular pressure; $\tau=$ time constant of isovolumetric relaxation; other abbreviations as in Figure 1.

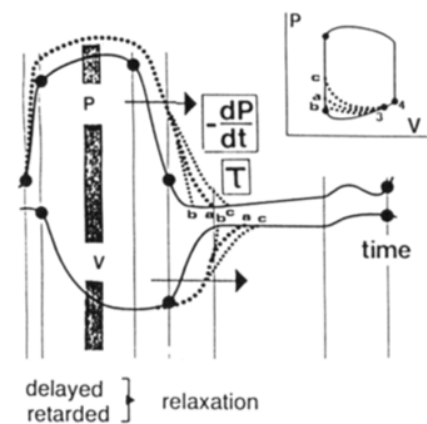

or

PROLONGED CONTRACTION

(physiologic) II

COMPENSATORY

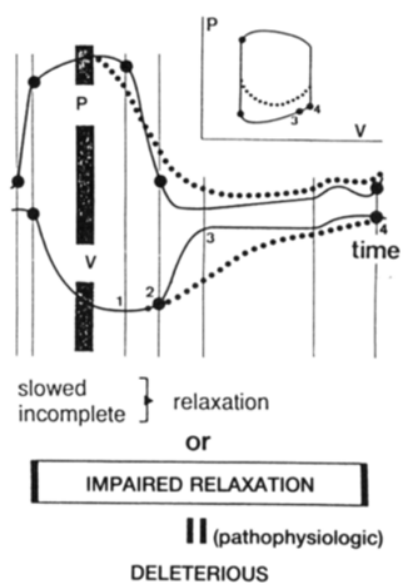

DELETERIOUS grounds and for practical diagnostic and therapeutic considerations. Whereas impaired systolic relaxation is purt of a pathophysiologic process that is always deleierious in the long run, prolonged contraction is physiologic, compensatory and not in itself deleterious.

Prolonged systolic contraction. The primary event in compensatory prolonged contraction is a delayed onset of relaxation, regardless of whether it is accompanied by physiologic changes in the rate of relaxation. An upward shift of the pressure-volume relation during true diastole is not observed in conditions of compensatory prolonged coniraction; an exception to this rule may be seen at inappropriately high heart rates. The causes of compensatory prolonged contraction (or delayed relaxation) are multiple (Fig. 3). A classical example of prolonged contraction is observed in conditions of acute or chronic systolic pressure or volume (over)loading, or both (Fig. 3, left). Similar changes in the systolic pressure curve pattern were illustrated in the early work by Frank in frogs and later by Starling in dogs (12). Ventricular hypertrophy, at least during the early phase, as well as modulation of ventricular performance by the coronary vascular (20-22) and endocardial (22-27) endothelium (Fig. 3, right) induce similar changes in the duration of systole. Substances such as angiotensin II, catecholamines (alpha,-agonist activity) and vasopressin, whose plasma levels may all be increased during overloading and in conditions of heart failure, elicit a similar response in systolic duration (25). As stated before, the mere prolongation of contraction can be accompanied by moderate (that is, physiologic) changes in the rate of contraction or relaxation. Physiologic modulation in the rate of contraction or relaxation will depend on sympathetic drive (28), plasma catecholamines (beta-adrenergic agonist activity) (29), instantaneous changes in load during the cardiac cycle (30) and frequency potentiation induced by changes in heart rate (29), among other factors.

Impaired systolic relaxation. By contrast, the primary event in impaired systolic relaxation is an inappropriately decreased rate or decreased extent of relaxation. As a consequence, relaxation extends into true diastole, with an upward shift of the pressure-volume relation. Ischemia and hypertrophy (at least in the advanced phase of hypertrophy), as in aortic stenosis or hypertensive heart disease, are common causes of impaired relaxation. Nonuniformity of ventricular performance, which often accompanies these two clinical conditions, may also constitute a cause of impaired relaxation regardless of the degree and extent of underlying ischemia or hypertrophy (31). In all of these clinical conditions, an inappropriately decreased rate or extent of relaxation may indeed result from disturbances in the so-called triple control of relaxation; that is, 1) from impaired activation-inactivation (calcium homeostasis, sarcoplasmic reticulum pump, contractile proteins); 2) from excessive changes in load; or 3) from inappropriate nonuniformities of load and activation-inaciivation in time and 


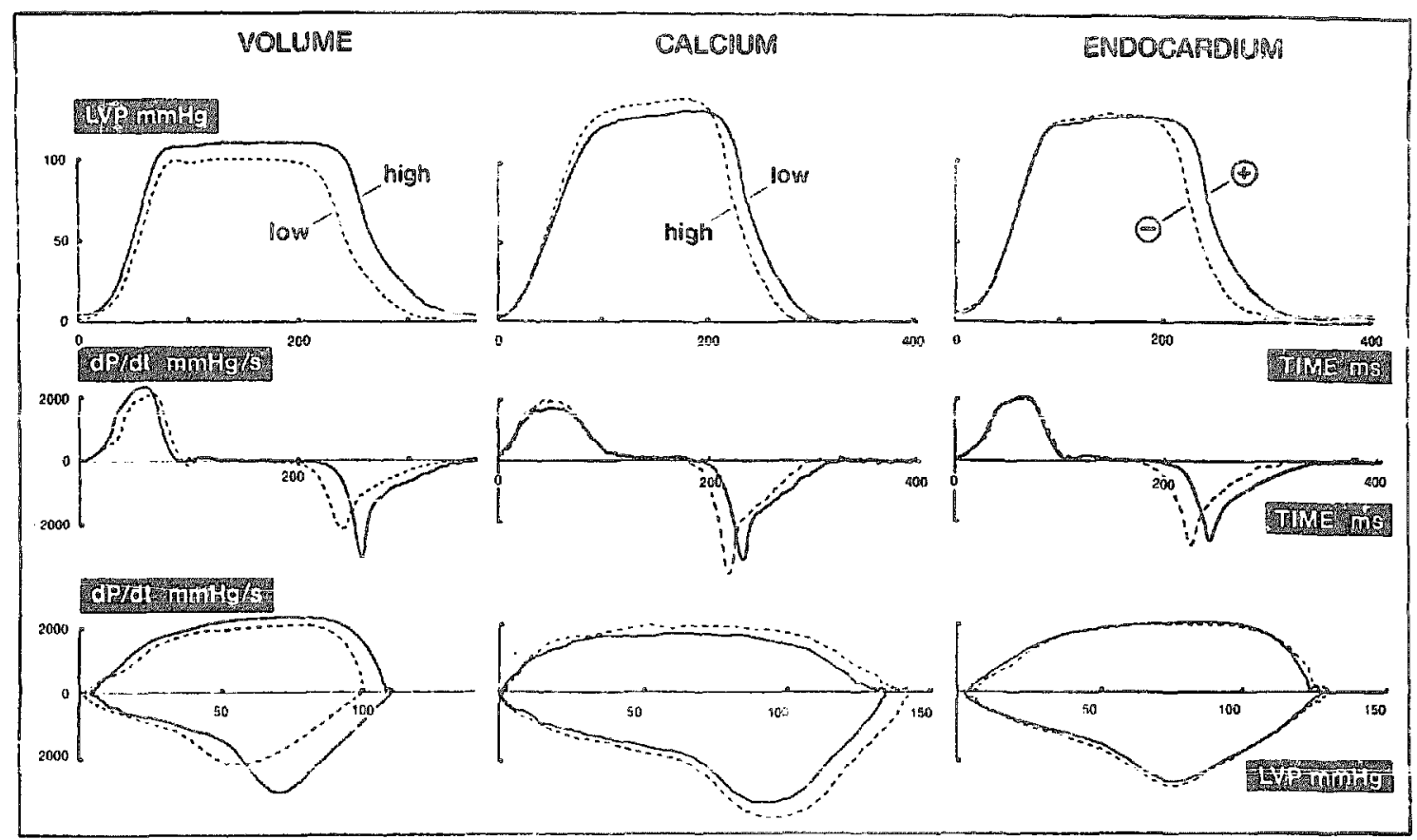

Figure 3. Effects of the three majur modulators of cardiac performance on systolic duration of pressure-time curves of canine left ventricle (22). For comparable changes in timing of relaxation, the rate ${ }_{\text {and }}$ pattern of relaxation were influenced differenty by the three rypes of modulation. Left ventricular pressurc (LVP) (top), first derivative of left ventricular pressure ( $\mathrm{dP} / \mathrm{dt}$;s. time, middule) and phase-plane (dP/dt vs. L.VP, bottom) tracings are displayed. Ir each panel, a bascline iracing (solid curve) and a tracing after intervention (dashed eurve) are superimposed. Left panels, Modulation of cardiac performance by changes in end-diastolic volume (heterometric autoregulation or Frank Starling mechanism). Compared with baseline (high), lowering left ventricular volume and pressure (low) incuced slightly lower rate of pressure increase and early onset of decrease in left ventricular pressure. As evident from the phase-plane tracing (bottom panel), initial decrease in left ventricular pressure and peak negative $\mathrm{dP} / \mathrm{dt}$ were slower at the lower end-diastolic volume, in contrast to the late decrease in left ventricular pressure, which was faster (i.e., projected below control iracing). Middulle paness, Modulation of cardiac performance by changes in contractility (homeometric autoregulation). Compared with baseline (low), intravenous administration of a small camount of alcium chloride $(0.05 \mathrm{mg} / \mathrm{kg}$ body weight; high) increased the rat of rise of pressure; left veniricular pressure was mildly increased. The oncet of the decrease in left ventricular pressure was either unchanged or occuried slightly earlier, as shown here. The rate of decrease in left ventricular pressure was increasec over its entire

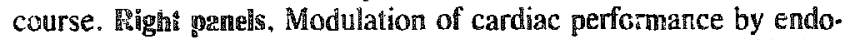
cardial endotheliun (endothelium-mediated autoregulation) (20-27). The endocardial endothelium was functionally inactivated by high power, high frequency intracavitary ultrasound $(-)$. Compared with baseline $(+)$, peak !efft ventricular pressure and peak positive $\mathrm{dP} / \mathrm{dit}$ remained unaltered. The decrease in left ventricular pressure was induced prematurely and revealed a slightly accelerated course, evidenced by a slight increase in peak negative $d P / d t$ and the projection of a decreasc in left ventricular pressure on the phaseplane tracing (botlorn panel) slightly below the baseline tracing. space. An extensive discussion of the triple control of relaxation has been published elsewhere (12).

Interaction of prolonged contraction and impaired relaxation. In many clinical conditions leading to diastolic failure, a clear distinction between the previous two features of the systolic contraction-relaxation cycle is difficult. Depending on a given disease and on the instant during the development of that disease, these two features can be present separately or in combination. This can be best illustrated by examining the pathophysiologic evolution of pressure or volume overloading, or buth, of the left ventricle (as in Fig. 4). Of importance is the general pattern of the pathophysiologic evolution with time and the paltern of the individual pressure curves in each cardiac cycle. In the evolution of pressure and volume overloading with time, several phases can be distinguished. The initial phrse is compensatory, with typical prolonged contraction and, hence, delayed or re- tarded relaxation; this may result from ventricular dilation (Frank-Starling mechanism), myocardial hyperirophy, increased plasma levels of various components (angiotensin, vasopressin), among other factors. In the second phase, inappropriately decreased rates of relaxation due to genuine impairment of relaxation may disturb the clinical picture. Along with decreased compliance and increased heart rate, impaired relaxation may result in an upward shift of the pressure-volume relation during diastole (Fig. 2, right), resulting in exercise intolerance. In the third phase of Figure 4, concomitant systolic failure develops. Systolic failure results in ioss of systolic compensation (that is, in decreased contractility during contraction and early ejection) and in loss of the capacity to prolong systole; instead, as in Figure 4 , the onset of relaxation is often induced prematurely which furtier complicates the already existing clinical picture of dias tolic failure. 


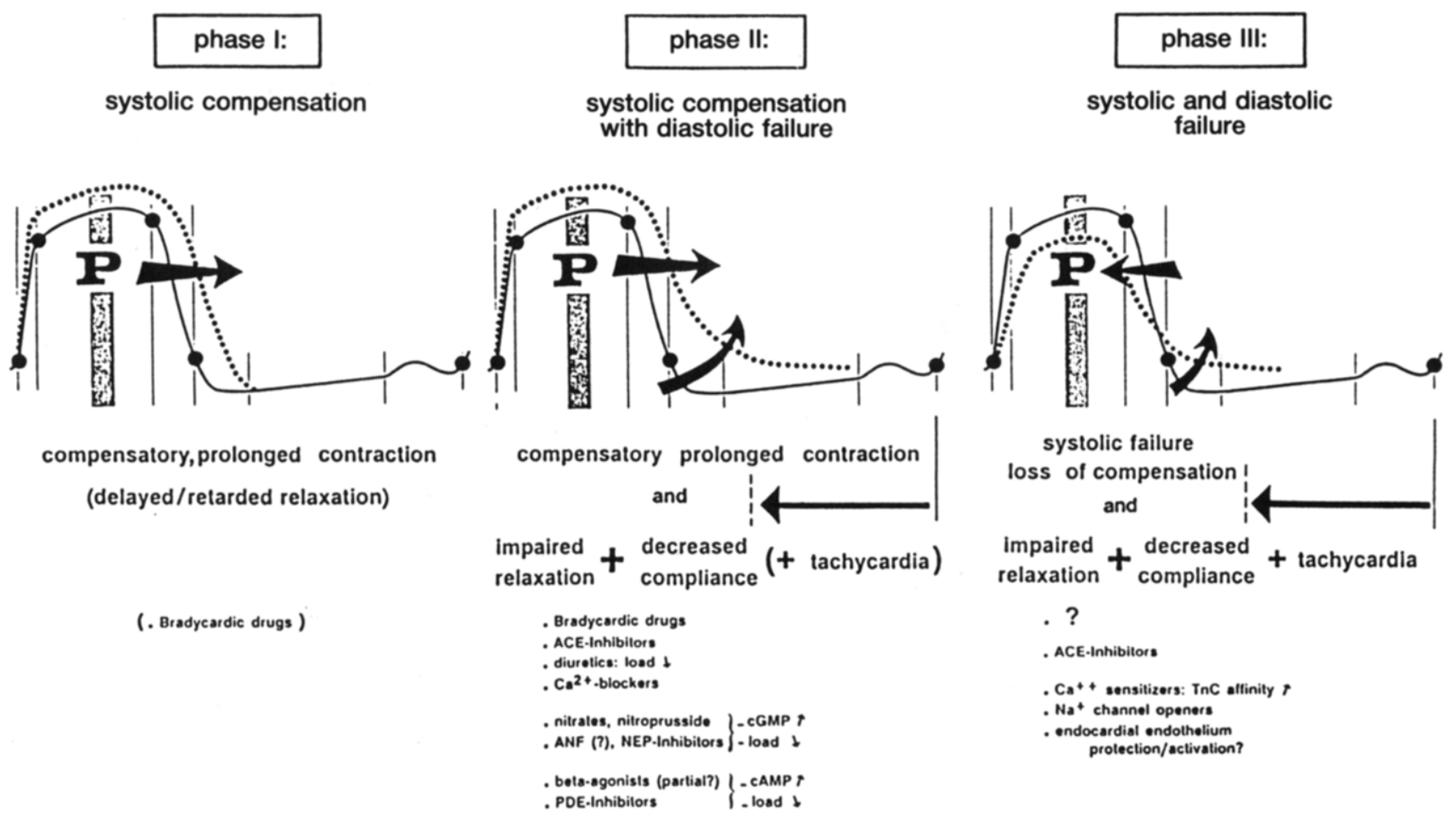

Figure 4. Successive phases during the pathophysiologic evolution of pressure or volume overloading, or both, of the left ventricle. Only pressure $(\mathrm{P})$ vs. time curves are shown. The $\mathrm{P}$ curve in full is the baseline $P$ curve before overloading. $A C E=$ angiotensinconverting enzyme; ANF = atrial natriuretic factor; $\mathrm{cAMP}=$ cyclic adenosine 5'-monophosphate; cGMP = cyclic guanosine 5'. monophosphate; NEP = neutral endopeptidase $\mathrm{PDE}=$ phosphodiesterase; $\mathrm{TnC}=$ troponin $\mathrm{C}$. In the data at bottom, $\lambda=$ increase and $y=$ decrease.

In patients with ischemic cardionyopathy, these three phases may, at a myocardial level and at any given time during the pathogenesis, develop simultaneously and interact with one another at the ventricular level. This may explain, at least in part, why diastolic dysfunction in different types of experimental and clinical myocardial ischemia, such as coronary occlusion, rapid pacing in the presence of coronary stenosis, multivessel coronary heart disease, reperfusion and stunning, may be rather complex and not eusily predictable.

Accordingly, although it is clear from the latter two examples of hypertrophic and ischemic cardiomyopathy that the causes of diastolic dysfunction and failure are multifactorial in most cardiac diseases (that is, based on a combination of the three major causes described [Fig. 1]), these pathophysiologic concepts should be kept in mind when developing a diagnostic and therapeutic strategy.

Implications for the evaluation of relaxation. Figures 3 and 4 illustrate that a full evaluation of systolic relaxation (that is, of isovolumetric pressure derline and early rapid filling), should encompass 1) measurements or indexes of tate of pressure decline and early rapid filling (peak negative
$\mathrm{dP} / \mathrm{dt}$, tau, isovolumetric relaxation time, early rapid filling rate); 2) measurements of the timing (normalized for heart rate) of the latter rate indexes; and 3) measurements of rate patterns, including early, middle and lare phases of the relaxation process. Simultaneous measurement of rate and timing may help to differentiate between conditions of prolonged contraction and of impaired relaxation. The need to examine rate patterns during the entire course of relaxation has been emphasized by us before (12). This point is also obvious from phase-plane $\mathrm{dP} / \mathrm{dt}$ versus pressure analysis (Fig. 3), where the rate of early pressure decrease, peak negative $\mathrm{dP} / \mathrm{dt}$ and rate of late pressure decrease are revealed as three distinct aspects of relaxation. In most cases, peak negative $\mathrm{dP} / \mathrm{dt}$ results from the transition from early to late rate of relaxation and may therefore not be representative for the entire process of relaxation. Moreover, early and late relaxation rates may be affected differently and may oîten change in opposite directions (30).

By extension, these measurements of systolic performance during relaxation should, in many clinical concitions or after various pharmacologic interventions (32), not necessarily be expected to correlate with measurements of diastolic function, such as diastolic pressure-volume relations.

\section{Implications for Therapy}

The rationale for treating patients with diastolic failure is to improve exercise tolerance by diminishing or suppressing upward shifts in the diastolic pressure-volume relation (Fig. 5). Although various drug regimens have been tested, 


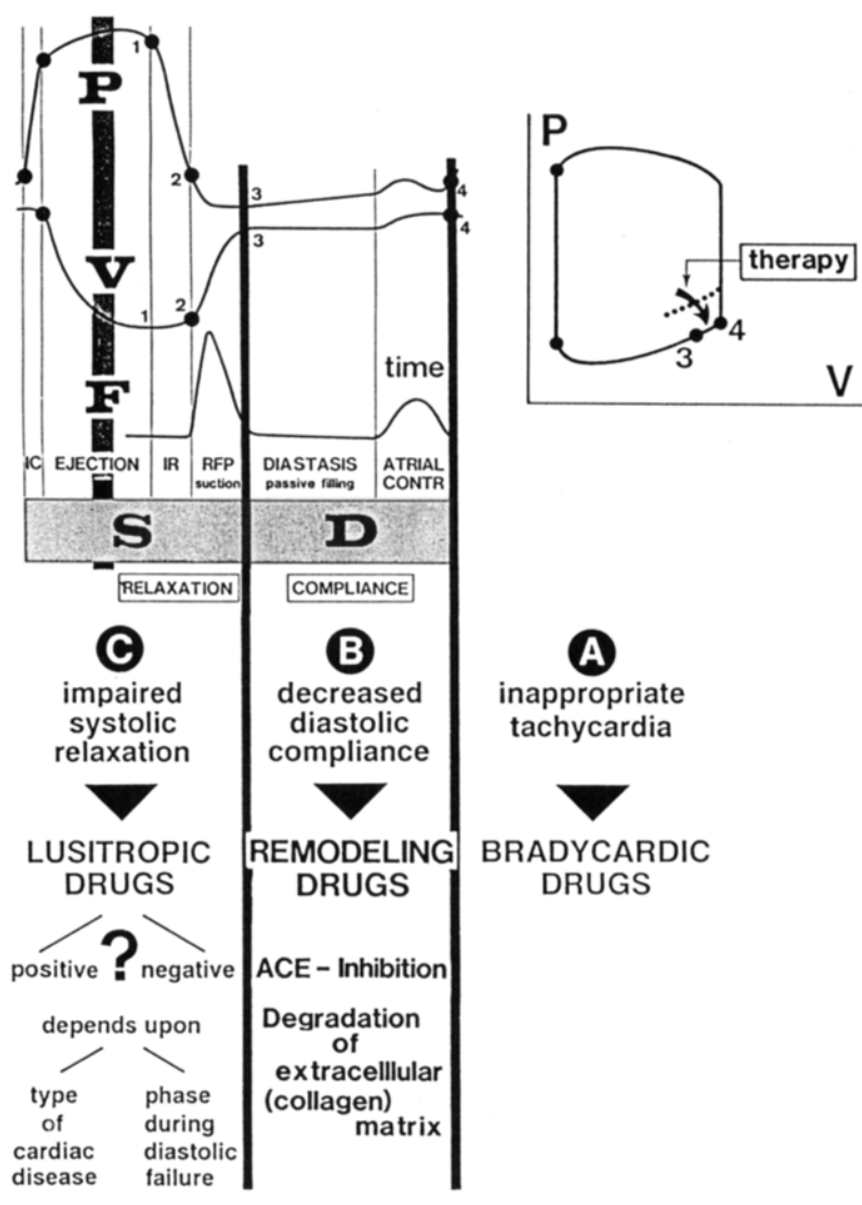

Figure 5. Classification of drug therapy for the treatment of diastolic failure. Abbreviations as in Figures 1 and 4.

no specific therapy is available. On theoretic grounds, and on the basis of the preceding classification of three causes, one could similarly conceive three classes of potential drugs.

When inappropriate heart rate is the major cause of diastolic failure, bradycardic drugs are the treatment of choice. Beta-adrenergic blocking agents, calcium channel blocking agents and, in specific conditions (such as atrial fibrillation), digitalis have all been successful in improving exercise tolerance due to primary diastolic failure. There have been several recent developments in such drugs. Some new molecules are potentially interesting with specific bradycardic properties but, unlike the other drugs of this type, are devoid of direct inotropic actions on the myocardium (33). Ideally, these drugs should preferentially suppress inappropriate increases in heart rate during exercise, with little or no effect on heart rate at rest.

Little attention has been directed toward the treatment of fundamental changes in myocardial compliance; however, the recent introduction of the concept of remodeling (34), in particular in the prevention of cardiac fibrosis and regression of hypertrophy, has emphasized the potential use of socalled remodeling drugs $(35,36)$. For example, various angiotensin-converting enzyme inhibitors and, more re- cently, antialdosterone agents appear so be most efficient as remodeling drugs $(35,37)$. Several other molecules aimed at degrading or digesting componerts, such as collagen 11 , in the extracellular matrix, are under development in an al tempt to optimize the ratio of the amount of collagen III to the amoumt of contractile proteins; however, the clinical applicability and benefits of these developinents reñain to be frmly established.

Most commonly used drugs for the treatment of diastolic failure belong to the category of so-called lusitropic drugs. Treatment with lusitropic drugs is aimed at improving impaired systobic relaration and is believed to help patients with diastolic failure, especially those with exercise intolerance. This class of drugs constitutes one of the most confusing and controversial issues in the field of cardiovascular therapy. Whereas the term /usitron;-* :s sufficiently useful to designate a relation to relaxatun and rapid filling, it is not specific enough to be useful as a basis for classifying negative and positive lusitropic drugs. Indeed, there are no positive and negativ e lusitropic drugs. Whether a given drug has a ncgaive or $\overline{\mathrm{A}}$ itive bitropic action will depend on hoth the type of $\mathrm{tl}$ : disease and the phase during the pathogenesis of the disease. For example, it follows from Figure 4, which shows the development of pressure or volume overloading in three successive phases, that treatment must be carefully selected to be appropriate for any one of the three phases. Similarly, in patients with ischemic cardiomyopathy, treatment of diastolic dysfunction and failure should be different, depending on the relative contribution of each one of these three phases on ventricular performance at any given moment.

Figure 4 also lists current potentially useful drugs for the different phases during ventricular overload. During the course of pressure or volume overloading with subsequent hypertroshic cardiomyopathy, angiotensin-converting enzyme inhibitors score high in all phases by their suppressive action on angiotensin $I I$ and on load and as remodeling drugs. Diuretic drugs consistently improve exercise tolerance with little change in systolic performance, probably directly through their effects on both right and left ventricular diastolic loading conditions as well as indirectly by reducing pericardial constraint on the left ventricle and on ventricular cross-talk $(10,38)$. Calcium channel blockers

*Lusitropic is derived from the Greek words $\lambda \cup \overline{0} \zeta, \dot{\eta}$, meaning loosing, releasing, ransoming, means of letting. deliverance from guilt, redemption of mortgage or pledge. emptying. evacuation. emission of semen, unraveling,

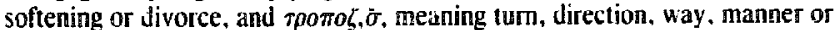
fashion. The term lusitropic was coined by Phylis B. Katz to mean predominantly acting (positively or regatively) on the relaxation of the heart both as muscle and as pump. The major advantage of the term lusiropic, (that is, its potcntial usefulness as a general term to describe interventions that preferentially act on relazation) unfortunately has the same limitations as line ferm inotropic for the contraction phase. Ir particular, it lacks sufficient specificity for the different phases to which the term is meant to relate during relaxation. Moreover. does it refer to changes in onset, in speed. in extent, or to changes in the ime pattern of relaxation with little changes in onset. in peak speed or extent, or a $t$ of these? Does it refer tc ivactivation. or does it also incorporate effects medited through changes in loading? (12). 
have been shown to improve exercise intolerance in phases I and II shown in Figure 4 for still largely unknown reasons (including relief of ischemia, decrease in intracellular calcium ion overload and improvement of inappropriate nonuniformities [39-42]) but are in our opinion expected to be deleterious in phase III. Nitrates, nitroprusside and related molecules have been shown to consistently decrease the diastolic pressure-volume relation $(43,44)$ and therefore to improve exercise tolerance (10). A diminished diastolic load of both the left and the righ; ventricle and decreased pericardial constraint have been invoked to explain this beneficial effect. Equally important but largely overlooked in all previous studies, however, is the direct effect of nitrates, nitroprusside and related molecules in abbreviating systolic duration, with no effect on contraction dynamics, by increasing myocardial intracellular cyclic guanosine $5^{\prime}$-monophosphate level (45). Such an effect could be particularly beneficial during phase II of the example shown in Figure 4. Similar to nitrates and nitroprusside, atrial natriuretic factor $(45,46)$ and neutral endopeptidase inhibitors, which inhibit the breakdown of atrial natriuretic factor, would at least theoretically also be expected to be benenicial in the treatment of diastolic failure. Partial beta-agonists may slightly shorten the duration of contraction, with little change in heart rate owirg to the concomitant beta-blocking properties. Such treatnient could be advantageous in phase II, where it would suppress excessive systolic compensatory prolongation while simultaneously accelerating the rate of relaxation. The latter drug actions would be deleterious, however, during monotherapy in the terminal phase of combined systolic and diastolic failure (47). For similar reasons, as well as for their vasodilator properties, phosphodiesterase inhibitors may also decrease the diastolic pressure-volume relations, thus improving exercise tolerance in patients with diastolic failure during phase II, but should be given with caution in phase III.

As explained before, in many diseases (for example, ischemic cardiomyopathy and, more conclusively, when in combination with pressure-overload hypertrophy [13]), the somewhat arbitrary but conceptualiy useful subdivision into the successive phases shown in Figure 4 is not always easily appreciated because various segments of the ventricular wall may undergo successive ph ases at different moments and at different speeds. Here, the efficacy of a drug will depend on the relative contribution of any one of these phases present in the various segments of the ventricular wall at any moment during pathogenesis.

Of interest, drugs with a positive or a negative lusitropic action in a given clinical setting will, in other conditions, not necessarily act as positive or negative lusitropic "drugs." Various calcium-sensitizing drugs (48), as well as sodium channel openers (49), have often, but erroneously, been referred to as negative lusitropic because of their contraction-prolonging effect. These cardiotonic drugs might indeed be less desirable in phase II of the example shown in Figure 4. During phase III, however, and in combination with angiotensin-converting enzyme inhibitors, for example, calcium or sodium sensitization might be potentially beneficial and, hence, be positive lusitropic. Conversely, partial betaagonists and calcium channel blockers might, as previously stated, be useful in phase II but not in phase III. In other words, there is no single standard lusitropic therapy for the treatment of diastolic failure.

\section{Conclusions}

In summary, although primary diastolic failure has been recognized as a distinct pathuphysiologic syndrome, an appropriate definition of this syndrome has long been lacking. Primary diastolic failure is due to an increased resistance to ventricular filling and results in an inappropriate upward shift of the diastolic pressure-volume relation, especially during exercise; this leads to exercise intolerance, with symptoms of congestion. The causes are known and are often multifactorial (i.e., inappropriate tachycardia, decreased diastolic compliance and impaired systolic relaxation). Pathophysiologic, impaired (incomplete or slowed) systolic relaxation must be conceptually distinguished from physiologic, compensatory prolonged contraction (delayed or retarded relaxation). Treatment of diastolic failure is feasible but necessitates a clear understanding of the etiology, pathogenesis and pathophysiology of the underiying cardiac disease. Optimal therapy will depend on the type of disease, on the phase during the pathophysiologic evolution of the disease and on the coexistence and relative contribution of various compensatory or decompensatory mechanisms. This often requires a comprehensive analysis of hemodynamics for example, with combined, echocardiographic and Doppler studies), compieted, whenever necessary, by catheterization, both at rest and during exercise.

\section{References}

I. Dougherty AH, Naccarelli GV, Gray EL, Hicks C, Gnldsteir RA. Congestive heart failure with normal systolic function. Am J Cardiol 1984;54:778-82.

2. Soufer R, Wohlgelernter D. Vita NA, et al. Intact systolic left ventricular function in clinical congestive heart failure. Am J Cardiol 1985;55:1032-7.

3. Cohn JN, Johnson G, and Veterans Administration Cooperative Study Group. Heart failure with normal ejection fraction. Cirsulation 1990;81: (suppl III)III-48-53.

4. Gaasch WH. Congestive heart failure in patients with normal left ventricular systolic function. a manifestation of diastolic dysfunction. Herz 1991;16:22-32.

5. Lorell BH. Significance of diastolic dysfunction of the heart. Annu Rev Med 1991;42:411-36.

6. Kupari M. Diastolic heart function and failure. J Int Med Res 1991;229: 479-81.

7. Manolas $\mathrm{J}$. Ischemic and congestive form of diastolic abnormalities during isometric stress assessed by handgrip-apexcardiographic test (HAT) (abstr). J Mol Cell Cardiol 1991;23(suppl V):V-78.

8. Shub C. Diastolic dysfunction: recognition and treatment. Highlights, The Heart House Learning Center. J Am Coll Cardiol 1992;7:7-12.

9. Quinones MA. Evaluation of diastolic function by echo/Doppler. Highlights, The Heart House Learning Center. J Am Coll Cardiol 1992;7:13-7 
10. Packer M. Abnormalities of diastolic function as a potential cause of exercise intolerance in chronic heart failure. Circulation $1990 ; 81$ (suppl III):III-78-86

11. Brogan WC, Hillis LD, Flores ED, inge RA. The natural history of isolated left ventricular diastolic dysfunt :on. Am \& Med 1992;92:627-30.

12. Brutsaert DL, Sys SU. Relaxation and distote of the heart. Physiol Rev 1989;69:1228-315.

13. Eberli FR, Apstein CS, Ngoy S, Lorell BH. Exacerbation of left vertricular ischemic diastolic dysfunction by pressure-overload hypertrophy. Circ Res 1992;70:931-43.

14. Chen C, Rodriguez L, Levine RA, Weyman AE, Thomas ID. Noninvasive measurement of the time constant of left ventricular relaxation using the continuous-wave Doppler velocity profile of mitral regurgitation. Circulation 1992;86:272-8.

15. Swynghedauw 8, Delcayre C, Cheav SL, Callens-ElAmrani F. Biological basis of diastolic dysfunction of the hypertensive heart. Eur Hean $J$ 1992;13(supp] D):2-8.

16. Kass DA, Wolff M, Maughan WL. Mechronism of exercise-induced elevations of ventricuiar ind-4tastolic prescure in human cardionyoputhy (abstr). Circulation 1992;86:1-514.

17. Tyberg JV, Misbach GA, Glantz SA, Moores WY, Parmley WW. A mechanism for shifts in the diastolic, left venericular, pressure volume curve: the role of the pericardium. Eur J Cardiol 1978;7(suppl):10́3-75.

18. LeWinter M, Pavelec $R$. influence of the pericardium on left ventricular end-diastolic pressure-segnuen! relations during early and late stages of experimental chronic volume overload in dogs. Circ Res 1982;50:501-9.

19. Ianicki IS. Influence of the pericardum and ventricular interdependence on left ventricular diastolic and systolic function in patients with heart failure. Circulation 1990;81(2):15-20.

20. Li K, Roulcau IL. Andrics 1.J. Brutsaert DL. Elfect of dysfunctional vascular endothelium on myocardial performance in isolated papillary muscles. Circ Res 1993;72:768-77.

21. McClellan G, Weisberg A, Kato NS, Ramaciotti R, Sharkey A, Winegrad $S$. Contractile proteins in myocardial cells are regulated by factor(s) released by blond vessels. Circ Res 1992;70:787-803.

22. Brutsaert DL. Endocardial and coronary endothelial control of cardiac performance. News thysiol Sci 1992 (in press).

23. Brutsaert DL, Meulemans AL, Siuido KR. Sys SU. Effects of damaging the endocardial surface on the mechanical performance of isolated cardiac muscle. Circ Res 1988;62:357-66.

24. Brutsaert DL. The endocardium. Annu Rev Physiol 1989;51:263-73.

25. Brutsaent DL. Andries LJ. The endocardial endothelium. Am J Physiol 1992;263:H985-H1002

26. Gillebert TC, De Hert SG, Andries LJ, Jagenau LJ, Brutsaert DL. Intracavitary ultrasound impairs left ventricular periormance: presumed role of endocardial endothelium. Am J Physiol 1992:263:H857-1H65.

27. De Hert SG. Gillebert TC, Brutsaer DL. Alteration of left ventricular endocardial function by intracavitary high-power ultrasound inieracts with volume, inotropic state, and $\alpha-\llbracket$ adrenergic stimulation. Circulation 1993;87:1275-85.

28. Henning RJ, Levy MN. Effects of autonomic nerve stimulation, asynchrony, and load on dP/dtmax and on dP/dimin. Am J Physiol 1991;260: H1290-H8.

29. Kambayashi M, Miura T, Oh BH, X̃ockmian HA, Müata K, Ross J. Enhancement of the force-frequency effect on myocardial contractility by adrenergic stimulation in conscious dogs. Circulation 1992;86:572-80.
20. Leite-Moreira AF, Gilleber TC. Load demendence in the intar heart (absit). I Am Coll Cardiol 1993;21:412A.

31. Brutsaert $D$ L. Nonunifominy: a physiologic modulator of contraction and relaration of the nomal heart. 3 Am Coll Cardiol 1987;9:341-8.

3.. Lang RM, Camoll DD, Nakanura S, Itoh H, Rajer SI. Role of adrenoceptors and dopanine receptors in nodulating left ventricular diastolic fuiction. Circ Res 1988;63:125-34.

33. Miura T, Miyazaki S, Guth BD. Kambayashi M, Ross J. Inflence of the force-frequency relation on left ventricular function during exercise in conscious dogs. Circulation 1992;86:563-71.

34. Weber KT. Cardiac interstitium in health and disease: the fibrillar collagen network. I Am Coll Cardiol 1989;13:1637-52.

35. Weber KT, Brilla CG. Pathological hypertrophy and cardiac interstitium: fibrosis and renin-angiotensin-aldosterone system. Circulation 1991;83: $1849-65$.

36. Brilla CG, Manicki IS, Weber KT. Impaired diastolic function and coronary reserve in genetic hypertension. role of interstitial fibrosis and medial thickening of intramyocardial coronary anteries. Circ Res 1991;67: 107-15.

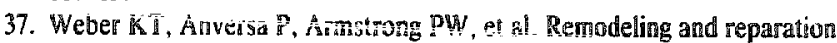
of the cardiovascular spstem. I Am Coll Cardiol 1992;20:3-16.

38. Wilson JR, Reichek N, Dunkman WB, Goldberg $\$$. Effect of diuresis on the performance of the failing left ventricle in man. Am Med 1981;70: 234-9.

39. Lorell BH. Paulus WJ, Grossman W, Wynne J, Cohn PF. Modification of abnormal left ventricular diastolic properties in patients with thypertrophic cardionyopathy. Circulation 1982;65:499-507.

40. Bonow RO, Rosing DR, Bacharach $\mathrm{SL}$, et al. Effects of verapamil on left ventricular systolic function and diastolic filling in patients with hyper. trophic cardiomyopathy. Circulation 1981;64:787-96.

41. Wunict PB, Courtois MR. Ludbrook PA. Efects of nifedioine on intrinsic myocardial stiffness in man. Circulation $j 986 ; \overline{74}: 13 \overline{3}-34$.

42. Walsh RA, O'Rourke RA. Direct and indirect effects of calcium entry blocking agents on isovolumic left ventricular relaxation in conscious dogs. J Clin Invest 1985;75:1426-34.

43. Brodie BR, Grossman W, Mann T, McLaurin L. Effects of sodium nitroprusside on left ventricular pressure-volume relations. I Cin Inves 1977;59:59-68.

44. Ludbrook PA, Byme JD. Kumik PB, Mcknight RC. Infuence of reduction of preload and afterlcad by nitroglycerin on left ventricular diastolic pressure-volume relations and relaxation in man. Circulation $1 ; ; 7 ; 56: 937-43$

45. Shah AM, Lewis MJ, Henderson AH. Effects of 8-bromr cyclic GRP va contraction and on inotronic response ci ferret cardiac unuscle. Mol Ceil Cardiol 1991:23:55-64.

46. Meulemans AL, Sipido KR. Sys SU, Brutsaert DL. Atriopeptin III induces early relaxation of ventricular cardiac muscle. Circ Res 1988;62: $1171-4$.

47. Xamotercl in Severe Heart Failure Study Group. Xamoterol in severe heart failure. Lancet 1990;2:1-6.

48. Ferroni $C$, Hano 0 , Ventura $C$, et al. A novel positive inotropic substance enhances contractility without increasing the $\mathrm{Ca}^{++}$transient in rat myocardium. J Mol Cell Cardiol 1991:23:325-31.

49. Cingolani HE, Wiedmann RT, Lvnch Jj, et al. Negative effect of DPI 201-106 and E4031. Possible role of prolonging action potential duration. J Mol Cell Cardiol 1990:22:1025-34. 